\title{
Finite Element Analysis as a response to frequently asked questions of machine tool mechanical design-engineers
}

\author{
Gerhard Kehl $^{1, a}$, David Blank ${ }^{1}$ and Christiane Höger-Riedel ${ }^{1}$ \\ ${ }^{1}$ University of Applied Sciences, Faculty of Engineering Management, Flandernstr. 101, Germany
}

\begin{abstract}
The finite element analysis (FEA) nowadays is indispensable in the product development of machining centres and production machinery for metal cutting processes. It enables extensive static, dynamic and thermal simulation of digital prototypes of machine tools before production start-up. But until now less reflection has been made about what are the most pressing questions to be answered in this application field, with the intention to align the modelling and simulation methods with substantial requirements. Based on 3D CAD geometry data for a modern machining centre (Deckel-Maho-Gildemeister DMG $635 \mathrm{~V}$ eco) merely the basic steps of a static analysis are reconstructed by FEA. Particularly the two most frequently asked questions by the design departments of machine tool manufacturers are discussed and highlighted. For this authentic simulation results are used, at which their selection is a consequence of long lasting experience in the industrial application of FEA in the design process chain. Noticing that such machine tools are mechatronic systems applying a considerable number of actuators, sensors and controllers in addition to mechanical structures, the answers to those core questions are required for design enhancement, to save costs and to improve the productivity and the quality of machined workpieces.
\end{abstract}

\section{Introduction}

The implied vertical machining centre (VMC) is a classical CNC machine tool for metal cutting. The shown VMC (Fig. 1) offers three Cartesian feed axes (X-, Y- and Z-axis) with a work space of $635 \mathrm{x}$ $510 \times 460 \mathrm{~mm}^{3}$ and is equipped with one milling spindle, cooling lubricant equipment and automatic chip conveyor.

Fig. 1 shows the supporting base structure of the examined machine with the arrows of movable feed axes. The machine bed rests on the ground via three elastic, damping and height adjustable levellers. The column is bolted down on the machine bed, composing the static machine frame, serving as a base for the automatic tool changer and the tool magazine. The workpiece table (X-axis) is located on the machine bed.

On the cutting tool side the linear movement in Y-direction is realized by the horizontal slide over the column. The vertical slide (Z-axis) is bounded on the front end of the horizontal slide and contains the spindle. To enable the linear motions accurately, each slide is guided by roller linear guides, driven by servo motor with belt-drive and attached ball screw.

\footnotetext{
${ }^{\text {a } C o r r e s p o n d i n g ~ a u t h o r ~: ~ g e r h a r d . k e h l @ h s-e s s l i n g e n . d e ~}$
} 


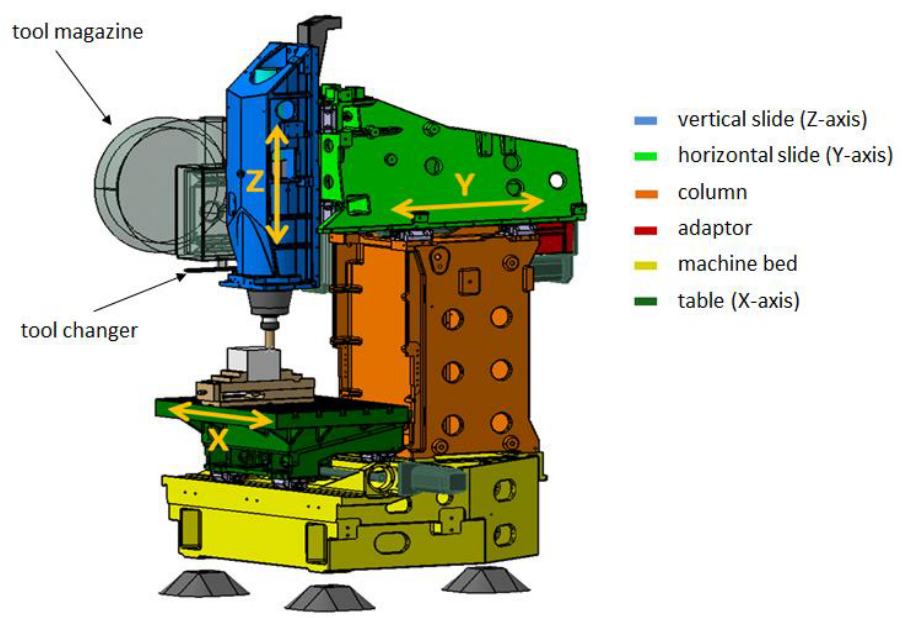

Figure 1. Axes configuration of VMC DMG $635 \mathrm{~V}$ eco.

\section{Import and preparation of 3D CAD Geometry Data}

U Machining process forces (from milling, boring, threading ...) at the tool centre point between tool (T) and workpiece (W) lead to displacements, which are caused by every participating machine component. The relevance of each machine component can be seen from the flux of force, which has to be determined at first.

Machine components with no influence on the deformation at the tool or the workpiece are suspended from the consideration and are not part of the supporting structure. Protective cover, chip conveyor, cooling fluid tank, control panel and service unit are famous examples for such peripheral components.

In terms of short FE modelling time as part of the design process chain it is recommended to take an up-to-date copy of the original CAD geometry data as basis and import a defeatured version of the supporting structure (as in Fig. 1) into the FEA software. For this purpose peripheral components have to be suppressed or deleted. Once the geometry is transferred to the FEA software material properties for each structural component are allocated dependent on the employed material (steel, grey cast iron, polymer concrete, brass ...). Typical analyses of machine tools are restricted to compliance behaviour and because of this the necessary geometry dimensioning of structural parts generates no strength of materials problems [1]. As a consequence only a few material properties are enough for such linear FEA models of machine tools, that is to say Young's modulus, Poisson ratio and material density.

\section{FE modelling of the machine tool}

The modelling process is divided into the volume meshing of the structural components (usually with parabolic hexahedral or tetrahedral finite elements) and the modelling of force transmitting machine elements by equivalent mechanical systems (linear spring, mass, viscous damper). This is relevant if modelling as volume is not reasonable or requires complex contact conditions. For the correct parameterization of equivalent mechanical systems (e.g. for bearings, ball screws, linear guides, flexible couplings, gears, machine levellers) measurement data usually is consulted, which can be delivered by notable suppliers or are common for experienced FEA engineers in the machine tool sector, gained by extensive calibration of experiment and simulation data [2].

For a general, non-customized FEA model it is suitable to represent cutting tool, workpiece and fixture as virtual rigid parts, e.g. the bar elements in Fig. 2. With this the points of load application at 
tool and workpiece can be linked to the machine structure and can be utilized as representative coordinates in the machines workspace.

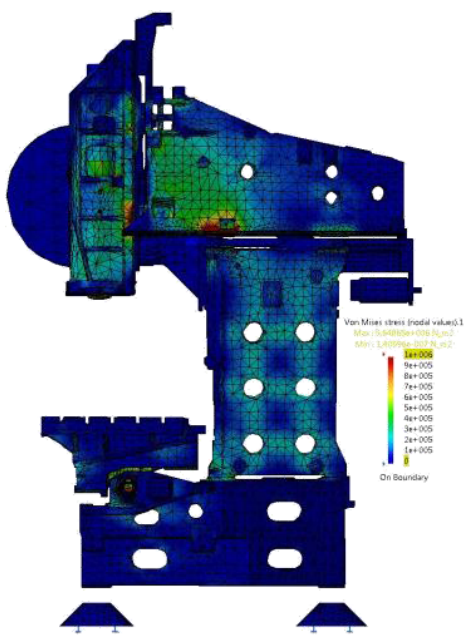

Figure 2. FE mesh of VMC with mechanical stress representation.

The FE model built up in the mentioned approach is the basis for answering typical questions of machine tool designers, which have to be solved in every machine tool design process.

\section{Frequently asked questions of machine tool design-engineers}

\section{"WHAT IS THE MOST SUITABLE GEOMETRY FOR MECHANICAL STRUCTURES LIKE MACHINE BEDS, COLUMNS ... ?"}

Obviously casted or welded metal components for VMCs should be configured at its best like space frame structures. This results from fundamental considerations about frame structures which should preferably possess a high degree of stiffness combined with low mass just like it is the case with constructions, bridges, antenna masts as well as with aerospace structures.

In this connection, bar triangles in contrast to bar rectangles are statically and kinematically determined inside and therefore possess a high level of stiffness as a result of the assumption that with flexible connection of the bars only tensile- and pressure loads are existent but no bending exposure [3]. Concerning the force $\mathrm{P}$ on point B, Fig. 3 demonstrates the comparably stiff characteristics of the framework consisting of triangular geometric basic element, here shown at the triangle $\mathrm{ABC}$ and ACD. In contrast to that, the rectangular bar composition $\mathrm{ABCD}$ (without the diagonal brace $\mathrm{AC}$ ) needs to be considered as flexible, so called four bar linkage. The fundamental difference in stiffness between both variants is obvious for flexible connections as well as for fixed connections of the bars.

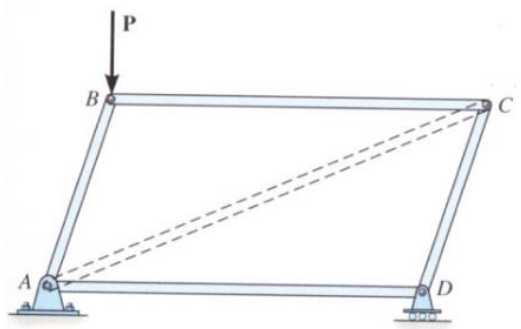

Figure 3. Geometric basic elements: triangle vs. rectangle [4]. 
Even though structure components from machine tools are usually casted, welded together, glued or screwed structures, the ideal geometric basic element always remains triangular.

Table 1 shows by means of one piece casted hollow blocks of similar mass and material, how stiffness can be increased through spatial ideal application of triangular basic elements (B) in comparison to the application of rectangular basic elements (A).

In all cases, the box shaped construction parts are unilateral rigidly mounted and the stiffness concerning bending and torsion are determined through simulative methods.

Table 1. Comparison of mechanical structures with rectangular and triangular base elements.

\begin{tabular}{|c|c|c|c|}
\hline & $\begin{array}{l}\text { improvement of } \\
\text { stiffness } \\
\text { stiffness B / } \\
\text { stiffness A }\end{array}$ & 48 & 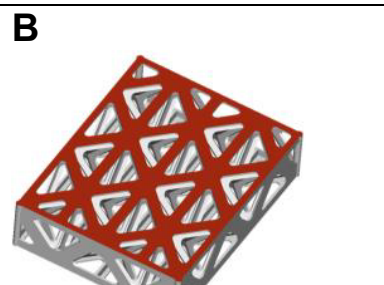 \\
\hline $\begin{array}{l}\text { bending } \\
\text { upwards }\end{array}$ & 1,53 & 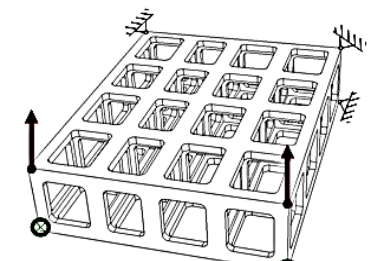 & 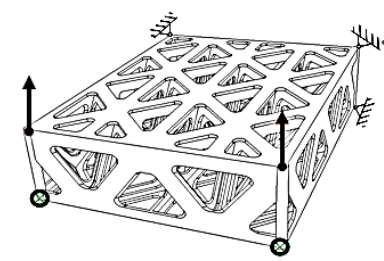 \\
\hline $\begin{array}{l}\text { bending } \\
\text { sidewards }\end{array}$ & 2,24 & 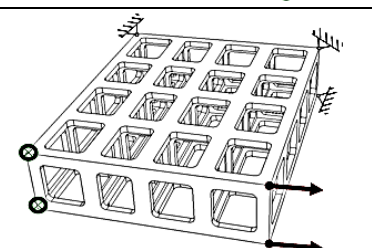 & 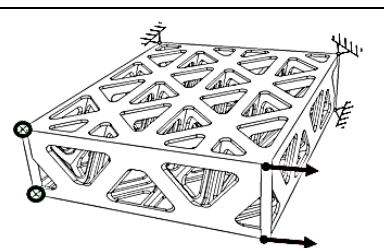 \\
\hline torsion & 3,95 & 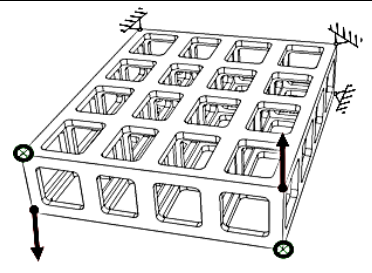 & 10 1 \\
\hline
\end{tabular}

The above presented improvements are admittedly seldom achieved in practice, because structure B usually cannot be implemented in real machines parts. The reasons are strong design restrictions (e.g. functional surfaces or contact areas to neighbouring parts have to be provided) or manufacturing restrictions (e.g. minimum and maximum wall thicknesses are given by the casting process). However, also improvements in stiffness by $10 \ldots 40 \%$ improve the machine tool and it's hereby produced workpieces significantly.

To illustrate that this supposed trivial design guideline even nowadays is not followed consequently, Fig. 4 shows two design states for a new machine bed. The revised design on the right side by applying triangular base elements has a $30 \%$ higher stiffness/mass ratio over the first design on the left side, which is based on rectangular geometry elements. As a consequence the machine bed with higher stiffness/mass ratio tends to result in better stability in cutting processes and higher quality of machined workpieces [5]. 

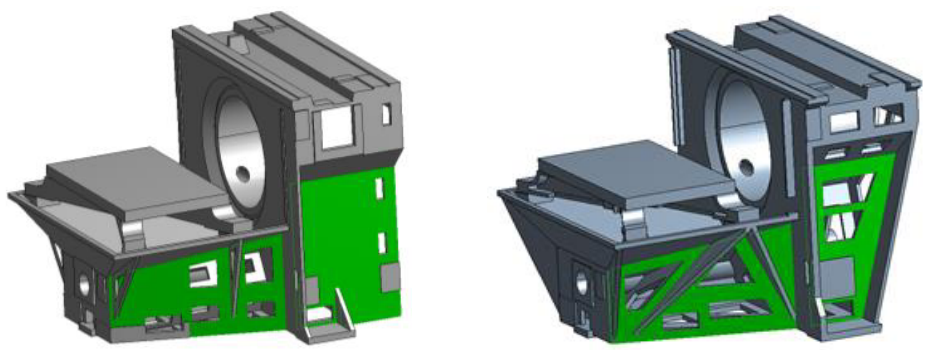

Figure 4. Two design states of a machine bed (mainly triangular/rectangular based).

Apart from generally applying triangular base elements the finding of load-capable geometry for machine structures can be done automatically by topology optimization, taking multiple load cases into account [6]. Topology optimization always utilizes the finite element analysis and leads to more detailed designs, but is not basically necessary, if the machine tool design-engineer in general tries to assemble mechanical structures by triangular base elements.

"DOES THE GRAVITY OF THE MOVING SLIDES ON THE STATIC MACHINE FRAME AFFECT THE STRAIGHTNESSES OF THE LINEAR FEED AXES ?"

In favour of analysing the feed axes straightness of the VMC a virtual precision test by FEA is suitable. For this purpose exclusively all horizontally moving slides are considered and represented by their masses. According to Fig. 2 those are the table with workpiece and fixture moving together horizontally in X-direction, whereas on the tool side the horizontal slide, the vertical slide with spindle and tool move together horizontally in Y-direction.

For comparison Fig. 5a shows the equivalent measurement setup for a precision test of a VMC in terms of the feed axes straightness. At this junction a dial indicator in the spindle is moved alongside a metal ruler mounted on the table surface tracing orthogonal deviations to the linear movement in the Y-direction.

Fig. 5b illustrates the emulated situation by FE simulation, in which only the movable masses in Y-direction and the opposing table are visible. The straightness error between tool and workpiece is calculated in defined intervals of the Y-axis stroke (e.g. every $50 \mathrm{~mm}$ ). For this static load cases under gravity of the movable masses are solved and the results are systematically interpreted.

The exemplary treated VMC shows straightness errors induced by self-loaded deformation primarily in the Y-axis. Fig. 6 outlines the orthogonal deviations in Z-direction over the Y-stroke for tool and workpiece and relatively between them $(\Delta \mathrm{Z})$. The latter reside with $\Delta \mathrm{Z} \approx 48 \mu \mathrm{m}$ in an inadmissible range, possibly leading to unreasonable straightness errors on machined workpiece surfaces. The dominant reason for this is the deformation of the overhanging Y-slide.

Upon detailed analysis of causes for this behaviour applicable design measures (e.g. stiffening the static machine frame and slides by adequate ribs, alternative linear guides) can be derived, improving the straightness of the Y-axis. Remaining straightness errors possibly may be compensated subsequently in the first place by providing uneven machining of the linear guide mounting surfaces during machine tool manufacturing or in the second place by using control algorithms during operation.

The machine foundation can also have remarkable influences on the straightness of the feed-axes, especially if the machine bed has poor inherent rigidity. However in this case the machine bed shows sufficient inherent rigidity leading to max. $10 \%$ straightness improvement, if the number of foundation points is increased significantly or if rigid levellers are used. 

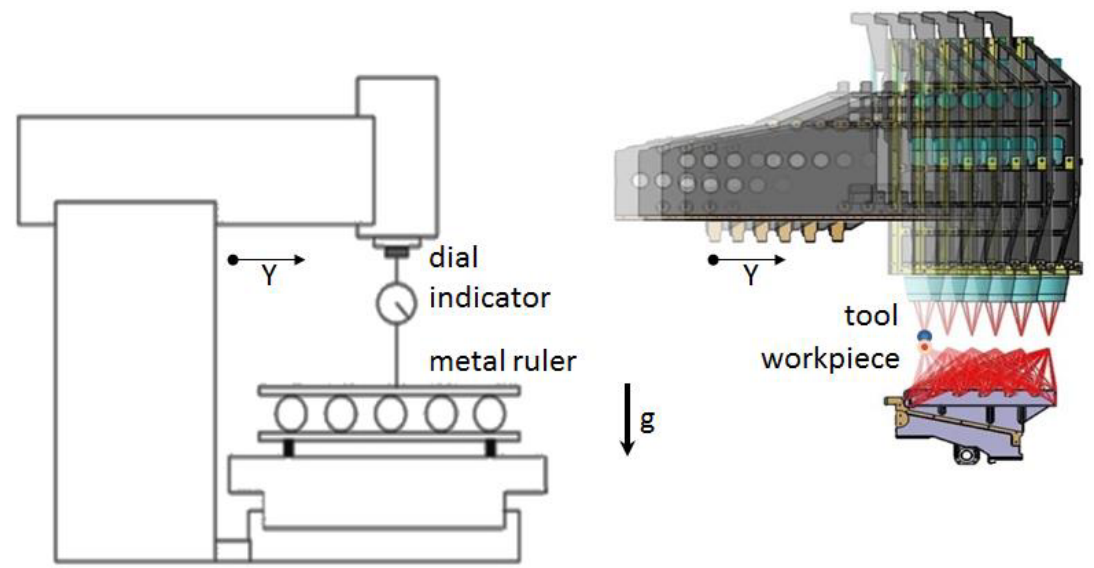

Figure 5. a) Measurement setup for precision test.

b) Simulation set up for precision test.

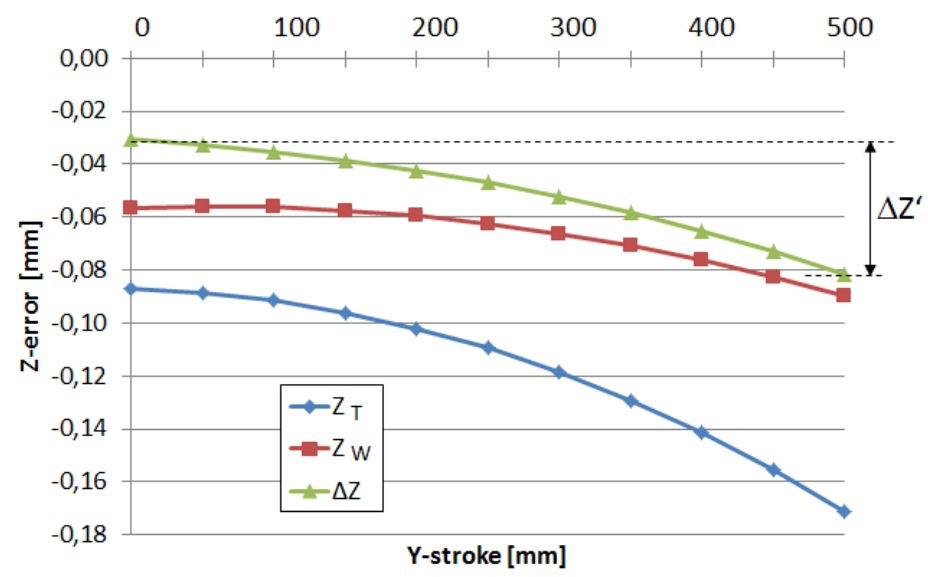

Figure 6. Straightness errors in Z-direction over Y-stroke.

\section{Conclusions}

The FEA is indispensable in the product development of machining centres and production machinery for metal cutting processes. Thus important questions, which occur during the design process, can be answered entirely targeting cost savings, improvement of productivity and high quality of machined workpieces.

\section{References}

1. M. Weck and C. Brecher, Fertigungssysteme 2, 7 (2002)

2. A. Rao, Factory and Plant Equipment. ANSYS Solutions - The Premier Magazine for Design Innovation 7, 4, pp.6-10 (2006)

3. K. Kabus, Mechanik und Festigkeitslehre, p. 52,7 (2013)

4. R.C. Hibbeler, Technische Mechanik 1, 12, p. 303 (2012)

5. P. Wagner and G. Kehl, "Will it Cut or Won't it?" - Crucial Question in Simulation-Aided Machine Tool Development, pp. 1-4 (CIM, Biograd, 2007)

6. G. Kehl, P. Jickeli, M. Schietinger, D. Blank, Topology Optimization of Rotating Automation Components for Machine Tools, pp. 208-217 (SIMULTECH, Colmar, 2015) 